\title{
CK One: a shared fragrance Corporeità e sessualità gender-free
}

\author{
Luca De Bortoli \\ Università di Bologna \\ luca.debortoli2@studio.unibo.it
}

\begin{abstract}
La polarizzazione maschile/femminile che aveva contraddistinto negli anni Ottanta la rappresentazione dei corpi e della sessualità all'interno del discorso della moda, tanto nell'individuazione di segmenti di mercato ("Pour homme"/"Pour femme"), che nel posizionamento semantico dei termini della categoria della sessualità, viene a cadere negli anni Novanta sotto la spinta di nuove proposte tendenti a una nuova concettualizzazione della sessualità più inclusiva (in linea con le proposte dell'allora neonata Queer Theory).

Si allinea in questo processo la campagna pubblicitaria del profumo Calvin Klein One, qui analizzata allo scopo di fornire un esempio paradigmatico di tale cambio di prospettiva legato ai temi della corporeità e della sessualità. Oltre a un'analisi semiotica dei vari elementi componenti la campagna, viene proposto un paragone in diacronia con alcune campagne precedenti della stessa fashion house; in questo modo, l'innovazione e lo spostamento da un paradigma separatista e stereotipizzante a uno inclusivo e neutralizzante sono ancora più evidenziati.
\end{abstract}

The polarization masculine/feminine which, in the 1980s, shaped the representation of sexuality and the body in the discourse of fashion - both in the delimitation of market segments ("Pour homme" / "Pour femme") and in the semantic positioning of the terms of the category of sexuality - dropped in the 1990s, under the impulse of new proposals that appealed to a new conceptualization of a more inclusive sexuality (in line with the newborn Queer Theory).

The advertising campaign for the fragrance Calvin Klein One exemplifies this process; it is hereby analyzed with the aim to provide a paradigmatic example of such a shift in perspective, concerning the themes of sexuality and body representation.

The analysis considers several aspects of the campaign and makes a diachronic comparison with other previous advertising campaigns from the same fashion house; thus, the innovation and shift from a separatist and stereotyping paradigm to a more inclusive and neutralizing one are even more highlighted.

\section{Parole chiave}

Sessualità, corporeità, Calvin Klein, profumo, moda

\section{Key Words}

Sexuality, body representation, Calvin Klein, fragrance, fashion

\section{Sommario}

1. Introduzione

1.1 Fragranze: appunti per una semiotica dell'olfatto

2. Analisi della campagna

2.1 "Soglie": flacone e packaging

2.2 Annunci stampa

2.3 Videoclip

3. Corpi e sessualità

3.1 Macho e Femme Fatale

3.2 Il caso Altered States

4. Conclusioni

Bibliografia 


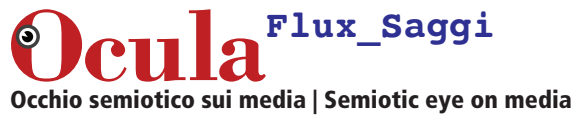

Luca De Bortoli | CK One: a shared fragrance. Corporeità e sessualità gender-free

\section{Introduzione}

Scopo di questo lavoro è la produzione di un'analisi semiotica della campagna pubblicitaria del profumo cK One della maison statunitense Calvin Klein, progettata e lanciata nel 1994, analizzando in particolare la rappresentazione dei corpi e lo spostamento nella categorizzazione della sessualità in prospettiva diacronica. CK One, infatti, pur non essendo stato il primo, è stato certamente il profumo unisex - l'azienda l'ha sempre definito una «shared fragrance» - che ha dato il via a questo trend nell'industria della moda e della cosmetica. Proporre un profumo di questo tipo è una scelta che a monte è dettata da un ripensamento della sessualità, non più separata in due poli (maschile e femminile) con conseguente target di mercato: rendere non pertinente la genderizzazione ha permesso di creare un profumo "inclusivo", per tutti. La condivisione e l'universalità sono i core-values della campagna, e sono stati interpreti anche della categoria della sessualità.

Conseguente a questa, anche la rappresentazione dei corpi ha avuto un ripensamento originale: se la polarizzazione tendeva all'iperbole attraverso stereotipi culturali (presentando modelli maschili virilizzati e modelle femminili sensualizzate), cK One ha saputo ricreare un'estetica senza pretese mitizzanti: ne è una prova l'impiego di modelli non professionisti, ritratti in situazioni comuni e in abbigliamento dallo stile dismesso e grunge. La possibilità di identificazione tra consumatore e modelli è un altro motivo di novità nel panorama della moda degli anni Novanta, che fino ad allora giocava sul desiderio dei consumatori del /voler essere/ come i soggetti rappresentati.

A ciò si lega anche la vicinanza e l'ispirazione del profumo alla cultura giovanile degli anni Novanta, alla generation $Y$ o generazione degli echo-boomers (v. Strauss e Howe 1991), votata alle cause civili (come l'ecologismo) e alla musica e cultura grunge.

Il successo della fragranza, che ha saputo sintetizzare in sé un'esigenza (quella del superamento del binarismo uomo/donna e della targetizzazione gender-based) e rendersi interprete di una comunità che poteva riconoscersi come depositaria di uno stile, è confermato anche dagli impressionanti dati di vendita: in soli 10 giorni dopo il lancio, il fatturato è stato di 5 milioni di dollari, mentre nel 1995 è stata la fragranza più venduta in assoluto. ${ }^{1}$

Nel corso degli anni, sono apparse numerose variazioni sul tema: oltre alle edizioni limitate Summer, le più note sono la versione $\mathrm{cK}$ Be, commercializ-

1 Da Women's Wear Daily del 7 ottobre 1994, cit. in: <http://www.nstperfume. com/2006/02/\%15/he-saysshe-says-calvin-klein-ck-one/> Ultima consultazione: 7 giugno 2017. 


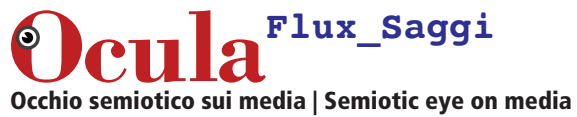

Luca De Bortoli | CK One: a shared fragrance. Corporeità e sessualità gender-free

zata a partire dal 1996 e la recente cK All (2017). Dopo più di un ventennio, la fragranza è ancora in produzione e riceve tutt'ora grande apprezzamento da parte dei consumatori.

\subsection{Fragranze: appunti per una semiotica dell'olfatto}

Prima di addentrarci nell'analisi della campagna pubblicitaria, vogliamo fare alcune considerazioni preliminari riguardo le fragranze in generale e $\mathrm{cK}$ One in particolare. Nella storia della semiotica molte sono state le semiotiche-oggetto che si sono differenziate, tra le altre cose, per la sostanza dell'espressione, come la semiotica del visibile, la semiotica dello spazio, la semiotica della musica, del gusto, ecc. ${ }^{2}$ L'olfatto è il senso più selvaggio e incontrollabile con cui ci misuriamo nell'esperire il reale, per via della sua imprevedibilità; esso ci garantisce una finestra sul mondo peculiare e differente dagli altri sensi: si tratta di una campionatura di una dimensione esperienziale continua (una materia - esattamente al pari dello spettro visivo o fonico), su cui, esercitando a dovere il naso e raffinando la sensibilità di discernere, è possibile arrivare a discretizzarne la materia per costruire un sistema (forma) relazionale.

Analogamente a quanto avviene, ad esempio, per un sommelier, il training per diventare un "naso" consente essenzialmente nell'acquisizione di una "griglia" di descrizione del dato, un sistema in grado di descrivere il continuum olfattivo. Sensi dell'olfatto più allenati e raffinati permetteranno di raggiungere distinzioni a grana sempre più fine. Il processo per ottenere questo risultato è duplice: una prima operazione consiste nella segmentizzazione della materia olfattiva attraverso alcune distinzioni di base (dell'ordine: /gradevole vs. sgradevole/, /floreale vs. speziato/, ecc.), in cui si tenta di rintracciare un sistema relazionale e differenziale, e in cui il valore di ogni unità possa emergere per differenza da quello delle altre. Sono suggestive le evidenti vicinanze con il metalinguaggio hjelmsleviano: l'operazione, infatti, è analoga alla trasformazione del purport in sostanza formata. La seconda operazione è una traduzione intersemiotica: ${ }^{3}$ dalla dimensione sensoriale olfattiva si passa a quella linguistica, ${ }^{4}$ fissando in parole uno spettro corrispondente di stimoli nervosi provocati da diverse sostanze.

2 Interessanti spunti sulla sensorialità e sulla loro importanza per la costruzione del significato ci vengono dati da Leroi-Gourhan (1977). Per la problematica specifica dell'olfatto e la sua importanza cognitiva, v. Cavalieri (2010).

3 V. Jakobson (1959) per il concetto di traduzione intersemiotica.

$4 \mathrm{Al}$ pari dei fonemi, questo rapporto è generalmente arbitrario. Infatti è sbagliato e riduttivo pensare che ogni nota (termine tecnico della profumeria che potremmo pensare come "fonema") sia l'emanazione o propagazione olfattiva di un frutto, spezia o altro materiale corrispondente. Questo in larga parte è vero, ma non in virtù di un'effettiva filiazione, bensì poiché chiamiamo allo stesso nome essenze e oggetti del mondo. Nel considerare le note, dunque, terremo presente che la nominazione avrà un carattere convenzionale e motivato, semmai, da vicinanze con fiori e piante del mondo naturale. Per esempio, la nota olfattiva 'speziato', è sì una nota di fondo corrispondente a spezie effettive da cui si propaga (quali cardamomo, pepe o cannella), ma la nota 'cuoio' racchiude invece betulla e laudano, che non hanno alcun rapporto di emanazione diretta con cuoio o pellami. 


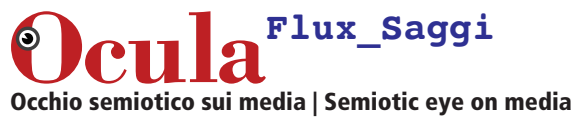

Luca De Bortoli | CK One: a shared fragrance. Corporeità e sessualità gender-free

Queste considerazioni non sono fatte per pedanteria accademica, ma hanno una funzione euristica precisa. Andando a considerare alcune descrizioni del profumo cK One, infatti, ci rendiamo conto che, per la stessa fragranza (per la sua composizione, o formula chimica ${ }^{5}$ ), le connotazioni linguistiche sono le stesse. Sembrerebbe, dunque, che precise composizioni olfattive siano legate ad altrettanto precise unità del contenuto. ${ }^{6}$

Per quanto riguarda cK One, tutte le descrizioni del profumo riportate in portali web specializzati in profumeria, anche in lingue diverse, ne rilevano il tratto di /freschezza/, e di /condivisione/ (per via del suo essere unisex). $\mathrm{Ne}$ forniamo di seguito alcune:

Two bodies, two minds, and two souls are merged into the heat and passion of one. This erotic cologne combines man and woman with one provocative scent. This clean, refreshing fragrance has notes of bergamot, cardamom, pineapple, papaya, amber, and green tea. ${ }^{7}$ (c.n.)

Pur-Frais-Léger. C'est une seule et unique senteur qu'elle partage avec lui, qu’il partage avec elle. Naturellement pur, simple et moderne avec une personnalité pleine de fraîcheur. ${ }^{8}$ (c.n.)

Iconica, unisex e moderna. La prima fragranza da condividere, $\mathrm{cK}$ One è un profumo universale, naturalmente pulito con un tocco rinfrescante. ${ }^{9}$ (c.n.)

Tutta la campagna, come vedremo, ha giocato moltissimo attorno al carattere di condivisione della fragranza tra uomo e donna, dovuto al fatto che que-

5 Si parla, in cosmesi, di piramide olfattiva; quella di cK One è composta come segue.

Note di testa: bergamotto, cardamomo, ananas, papaya, green tree accord.

Note di cuore: hedione high-cis (derivato del gelsomino), violetta, rosa, noce moscata, green tree accord.

Note di base: muschio, ambra, green tree accord.

La ricostruzione della piramide - per suggestionarci di nuovo con Hjelmslev e la fonologia praghese, notiamo che l'operazione è del tutto analoga all'individuazione dei femi - avviene seguendo il pioneristico Perfumes, Cosmetics and Soaps (1923) di W. A. Poucher, chimico amatoriale. La sua proposta è l'individuazione di classi di note in base alla velocità della loro evaporazione e a una somiglianza tra profumi (ad esempio, la famiglia delle note 'orientali' comprende patchouli, cannella, vaniglia).

6 È tutto da discutere quanto questo legame sia frutto di considerazioni culturali o di predisposizioni biologiche; è risaputo, ad esempio, che nel regno animale l'olfatto serve a riconoscere alimenti velenosi, mentre, al contrario, ci sono profumi fortemente culturalizzati. Per alcune culture, ad esempio, l'incenso ha una specifica funzione religiosa e rituale, mentre per altre è semplicemente ornamentale.

7 Tratto da FragranceX.com (<https://www.fragrancex.com/products/_cid_ perfume-am-lid_c-am-pid_104w__products.html >). Ultima consultazione: 10 giugno 2017.

8 Tratto da Galeries Lafayette.com (<https://www.galerieslafayette.com/p/ ck+one+-+eau+de+toilette-calvin+klein/82610858/378>). Ultima consultazione: 10 giugno 2017.

9 Tratto da Limoni.it (<https://www.limoni.it/cK-one-001076100ocf.html>). Ultima consultazione: 10 giugno 2017. 


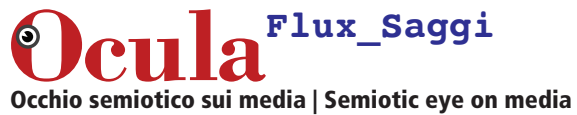

Luca De Bortoli | CK One: a shared fragrance. Corporeità e sessualità gender-free

sta distinzione non viene più ad essere pertinente. Se, in realtà, certe descrizioni possono essere state altamente influenzate dagli slogan e dalla headline della campagna (come la descrizione tratta da FragranceX: «Two bodies, two minds, and two souls are merged into the heat and passion of one»), è vero che il carattere di neutralità e di freschezza sembrano essere propri della fragranza. ${ }^{10} \mathrm{Ci}$ sono note tradizionalmente e culturalmente associate alla femminilità (come le note floreali) o alla mascolinità (cuoio e speziate). Il proporre un bouquet di note percepite come neutre o fresche è un espediente congeniale per fare tabula rasa nella tavolozza delle essenze, proponendone una universale e per tutti. Lo stesso procedimento, vedremo, è stato adottato per spazzar via alcuni stilemi e stereotipi di rappresentazione del corpo femminile e maschile in voga negli anni Ottanta, proponendo una rappresentazione della sessualità neutralizzata.

\section{Analisi della campagna}

La campagna si presenta globalmente come molto coerente e ben strutturata, in cui ogni componente è come una variante sul tema, rappresentando una declinazione dei medesimi valori; sembra lecito pensare, quindi, che ci sia a monte una consapevole programmazione semiotica (o almeno ispirata ai principi del marketing ${ }^{11}$ ). La campagna si compone principalmente di annunci stampa e videoclip; elementi essenziali sono tuttavia anche il packaging e il flacone, che interpretano a loro modo i forti core-values, che analizzeremo di seguito.

\section{1. "Soglie": flacone e packaging}

La fragranza è contenuta in due contenitori, uno inglobato nell'altro: il primo è ovviamente il flacone, a sua volta contenuto nel packaging in cui viene commercializzato (v. fig. 2.1). Da un punto di vista semiotico, possiamo considerare questi elementi come delle interfacce ${ }^{12}$ necessarie al consumatore per entrare in contatto con il profumo. Al pari del paratesto, non si tratta di uno spazio neutrale, avente una semplice funzione pratica (ossia, contenere e trasportare il prodotto); al contrario, in questi elementi possiamo rintracciare specifiche marche enunciazionali, molto importanti nell'economia generale della campagna e del suo senso.

- Flacone. Il flacone, da un lato, è minimalista e votato alla neutralità: si presenta come una bottiglia in vetro satinato su cui, come nel packaging, sono impressi solo il logotipo e il nome del brand. La forma è semplice ed

10 E, nello specifico, dovuti alle forti note agrumate.

11 Del resto, Calvin Klein, nel 2004, si rivolse all'agenzia The Semiotic Alliance per l'ideazione della campagna per il profumo Euphoria, divenuto un caso memorabile per i profitti miliardari. L'azienda, come afferma sul sito, utilizza e applica strumenti semiotici avanzati per i brand e il marketing. (Cfr.: <http://www.semiotics.co.uk/p/5/ calvin-klein-s-euphoria>). Ultima consultazione: 16 giugno 2017.

12 "Soglie" direbbe forse Genette. 


\section{Dcula \\ Flux_Saggi \\ Occhio semiotico sui media | Semiotic eye on media}

Luca De Bortoli | CK One: a shared fragrance. Corporeità e sessualità gender-free

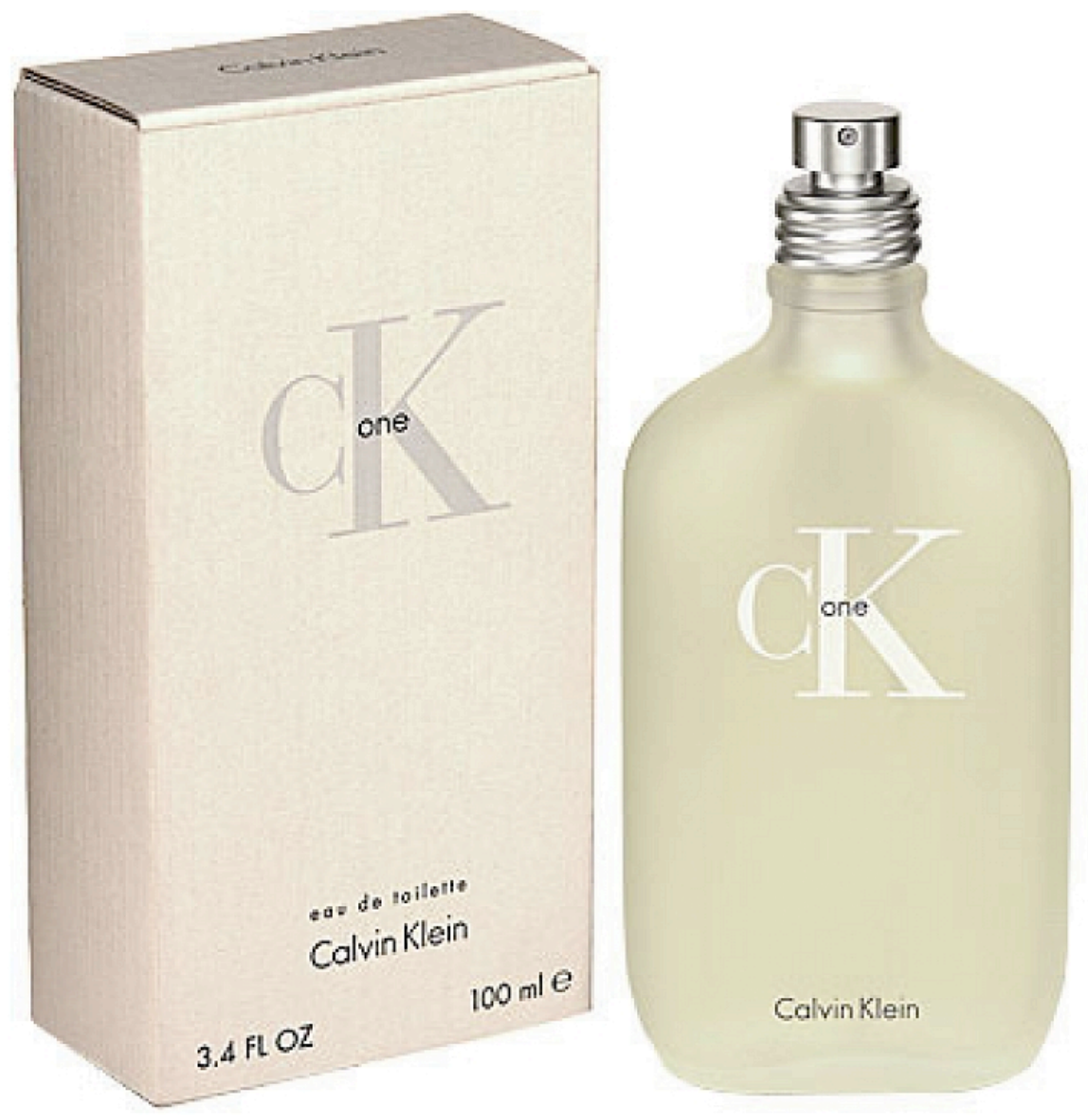

Figura 2.1. Packaging e flacone.

essenziale, i contorni sono puliti, il colore del vetro è algido e puro. Tutto è votato all'assenza di connotazioni, nel tentativo di ricreare una universalità virtuale, che ognuno possa in seguito interpretare a proprio modo. Tuttavia, potremmo fare un'osservazione di carattere storico-culturale: la somiglianza del flacone con una bottiglia di whiskey o di altri superalcolici (specialmente per il tappo in alluminio) attivano quella parte di enciclopedia locale che ha per tema la cultura grunge, movimento tipicamente giovanile e provocatore diffuso negli Usa nei primi anni Novanta. Questa osservazione è stata avanzata anche da Lizzie Ostrom nel suo Perfume: a Century of Scents: la sezione dedicata a cK One reca il sottotitolo «The Grunge Perfume», e così si dice riguardo al flacone: «the intimation was that these models were all sharing a flacon of CK One between them in the same way they might pass about the (very similar-looking) bottle of Absolut vodka» (Ostrom 2011: 96). 
Luca De Bortoli | CK One: a shared fragrance. Corporeità e sessualità gender-free
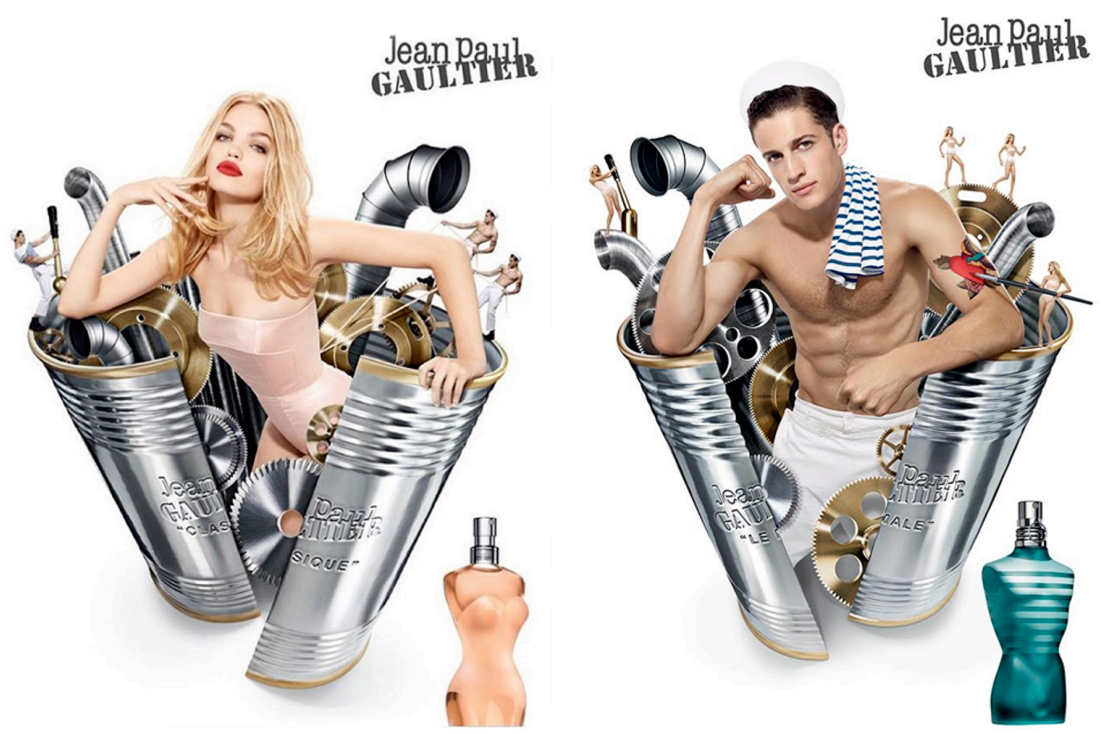

Figura 2.2. Annunci stampa per Classique $e$ Le Male di Jean-Paul Gaultier

- Packaging. Ideato da Fabien Baron ${ }^{13}$ il packaging che racchiude il flacone è una scatola di cartone, anch'essa molto minimale. Sono impressi nella confezione solo il nome/logo del profumo e il nome della maison. Il cartone utilizzato è riciclato e riciclabile, ed è piegato ingegnosamente senza l'utilizzo di colla o altri materiali chimici. Si tratta di una scelta piuttosto insolita per l'industria cosmetica, in cui, in genere, si utilizzano anche elementi secondari e di involucro per ribadire i propri values e la propria brand-identity, ${ }^{14}$ ma coerente con il senso complessivo della campagna: nel caso di cK One, il processo è avvenuto per sottrazione e il packaging è minimalista per evitare di aggiungere connotazioni all'ideale di /neutralità/ e purezza che il profumo (e la campagna) recano in sé. L'unico discorso che possiamo rintracciare è quello ecologista (materiali riciclati e riciclabili, e nessun impiego di colla), che risente (come la cultura grunge) del clima votato alle cause civili dei primi anni Novanta. Come vedremo nell'analisi degli annunci stampa, Calvin Klein ha espressamente voluto ricreare un'estetica non-mitizzata, senza pretese estetizzanti e vicina al mondo "reale", in cui fosse possibile riconoscersi: una sua fonte di ispirazione è stata la comunità della figlia e dei suoi amici, vicini alla cultura grunge.

Dunque, non solo per la fragranza in sé (in cui prevale l'utilizzo di note "neutre", unisex e che la rendono condivisibile), ma anche per gli elemen-

13 Baron realizzerà anche l'intera campagna pubblicitaria per il rilancio della linea allargata cK One (dal 2011), che comprende ora anche una linea di jeans e di intimo. Cfr.: <http://baron-baron.com/digital-cK-one/> (Ultima consultazione: 19 maggio 2017).

$14 \mathrm{Si}$ pensi, ad esempio, alle borse di Harrods, o ai pacchetti color turchese di Tiffany, elementi accessori, ma che hanno una specifica funzione di riconoscimento del brand, contribuendo a ribadirne l'estetica. 


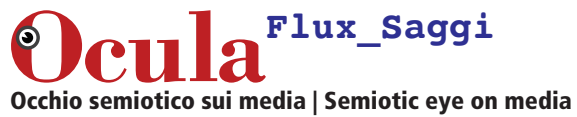

Luca De Bortoli | CK One: a shared fragrance. Corporeità e sessualità gender-free

ti in cui è contenuta (flacone e packaging), $\mathrm{cK}$ One rappresenta un punto di rottura con l'industria cosmetica, abituata a lavorare sul doppio binario del pour homme e pour femme. Un altro elemento di rottura è la trasgressione di una regola non-scritta della comunicazione pubblicitaria di profumi, un tropo ricorrentissimo: l'identificazione (esplicita o accennata) tra la boccetta del profumo e il testimonial dell'annuncio pubblicitario. Casi famosi sono ad esempio J'adore di Christian Dior, Classique e Le Male di Jean Paul Gaultier (v. fig. 2.2), Candy di Prada; analoghe e frequenti similitudini sono quelle che vogliono rimarcare anche il nome del profumo con la forma della boccetta: Trésor di Lancôme, ad esempio, ha la forma di un diamante, Flower di Kenzo ha la forma allungata di un papavero, 1 Million di Paco Rabanne ha la forma di un lingotto.

Per quanto riguarda cK One, il secondo tipo di similitudine (nome-flacone) è piuttosto impossibile da produrre: come esprimere il concetto di "One" (che sia "monade", "unità", "universalità", "Uno di Plotino", ecc.)?

Anche il primo tipo di similitudine (flacone-testimonial) non è individuabile, non essendoci un modello predominante sugli altri. Certamente Kate Moss e Jenni Shimizu sono tra quelle che compaiono con più predominanza negli annunci (v. 2.2), ma è errato definirle 'testimonial': non sono mai sole assieme al flacone, né è possibile trovare analogie plastiche con esse. Inoltre, la similitudine generalmente sembra essere subordinata al sesso del modello: si danno forme arrotondate e dolci per le fragranze femminili, squadrate per quelle maschili.

CK One è invece svincolato da questa logica, che più in generale coinvolge un tipo determinato di corpo, poiché, nell'ottica della campagna, la rappresentazione dei corpi non passa attraverso il filtro di una distinzione sessuale polarizzata su due termini (femminile/maschile). CK One è universale, e per mantenere la sua virtualità non può "attualizzarsi" in un corpo specifico.

\subsection{Annunci stampa}

L'annuncio a stampa originale del profumo è quello visibile in figura 2.3, assieme alle varianti delle figure 2.4 e 2.5, ${ }^{15}$ opera di Steven Meisel. Meisel è un fotografo e artista controverso, che proprio per Calvin Klein ha realizzato campagne che hanno soventemente sollevato l'opinione pubblica. La rappresentazione dei corpi di Meisel spesso riprende una sessualità in qualche modo "malata": nel 1993, ad esempio, grande scalpore fece la campagna $\mathrm{cK}$ Underwear, capostipite di un sottostile detto heroin-chic, ${ }^{16}$ che ritraeva una Kate Moss scheletrica, emaciata, con occhiaie scavate proprio come si trat-

15 Tratte da: <https://explore.calvinklein.com/en_US/timeline/1994/4/116-cKone-calvin-klein>. Ultima consultazione: 24 giugno 2017.

16 Non da sottovalutare l'uscita, nello stesso tempo, di iconici film legati alla tossicodipendenza (in particolare da eroina), quali Kids (Larry Clark, 1995) o Trainspotting (Danny Boyle, 1996), così come il suicidio di Kurt Cobain (1994). Il trend heroin-chic ha fatto da isotopia nella moda fino alla fine degli anni Novanta, legato a doppio taglio alla polemica sull'anoressia. 


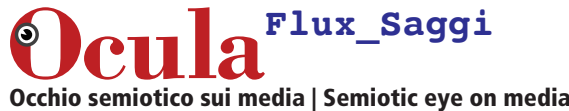

Luca De Bortoli | CK One: a shared fragrance. Corporeità e sessualità gender-free

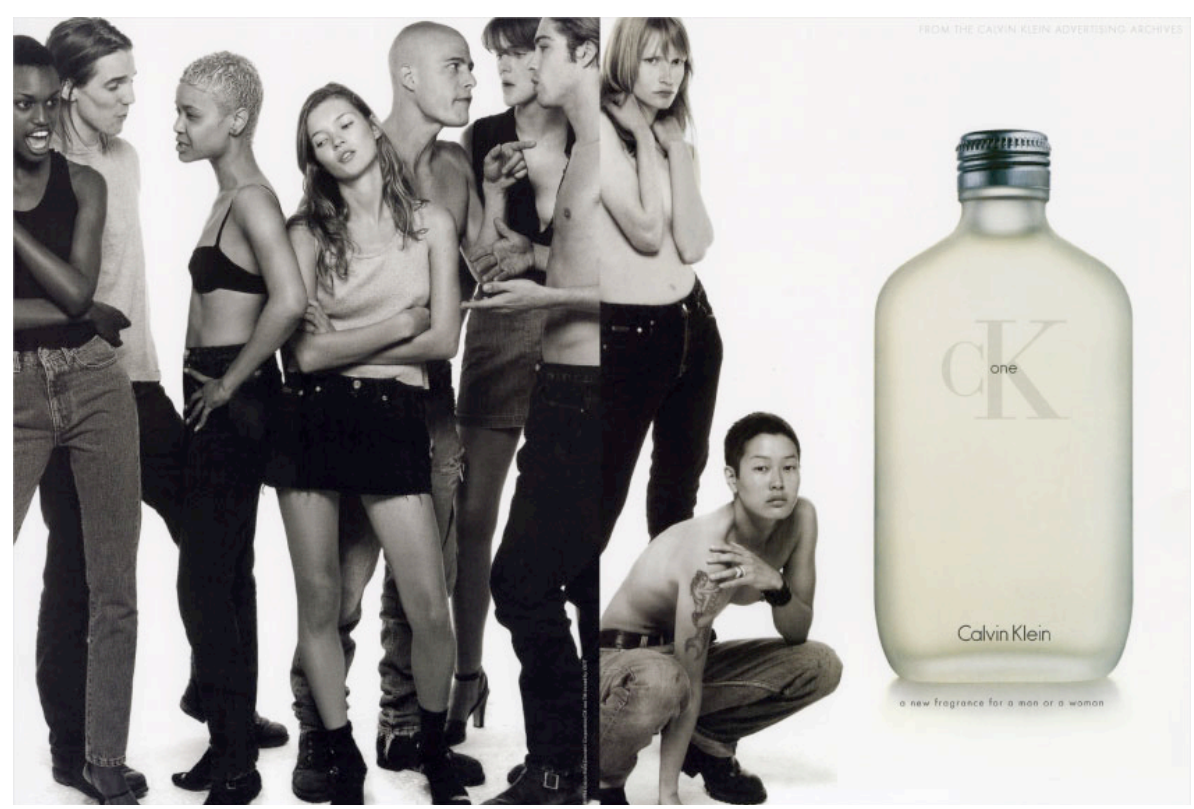

Figura 2.3. Annuncio stampa per cK One. (C) S. Meisel, 1994.

tasse di un'eroinomane. Due anni più tardi si parlò pure di kiddie porn ${ }^{17}$ per la campagna cK Jeans, girata in quello che sembrava lo scantinato di un pedofilo.

L'estetica di Meisel ricerca una bellezza diversa, e proprio nella rappresentazione dei corpi che viene fatta per la campagna di cK One notiamo una grande rottura con gli stilemi che hanno accompagnato la moda negli anni Ottanta. Tutti gli annunci presentano la medesima suddivisione in due parti nettamente separate tra loro (tanto che il corpo dei modelli che si trovano al limite tra i due è visibilmente tagliato), analogamente a quanto avviene per la successione di tableaux del videoclip (v. 2.3). Con l'eccezione del packshot, che ha un viraggio cromatico tendente al grigio/giallo paglierino, le fotografie sono realizzate in bianco e nero. La scena ritratta nella parte sinistra è dinamica, e raffigura un gruppo di ragazzi dallo stile urban/grunge e underground intenti a discutere (sembrerebbe piuttosto animatamente) fra loro. Con l'eccezione di alcune paia di scarpe femminili, di un reggiseno (sfoggiato però a mo' di canotta) e di due gonne (comunque di jeans), l'abbigliamento che i modelli indossano è assolutamente interscambiabile tra i modelli rappresentati, componendosi praticamente di jeans e magliette bianche o nere: il loro stile è il medesimo, ispirato, ancora, a un'estetica grunge. Una prova di commutazione non rivelerebbe differenze significative se al posto dei modelli ci fossero modelle e viceversa; da notare, inoltre, che molti ragazzi hanno i capelli lunghi, e molte ragazze li hanno corti o quasi a zero. La parte destra degli annunci si

17 Il videoclip consisteva in una serie di interviste a giovani modelli che rispondevano a domande fuori campo di una voce maschile. L’indignazione generale era causata dal fatto che questo set sembrava imitare dei provini per un film pornografico di bassa lega o per video erotici autoprodotti, e per di più con dei teenager. L'FBI aprì un fascicolo di investigazione, e pure Bill Clinton (allora presidente), intervenne sul caso condannando duramente le scelte estetiche della fashion house. 


\section{Ocula \\ Occhio semiotico sui media | Semiotic eye on media}

Luca De Bortoli | CK One: a shared fragrance. Corporeità e sessualità gender-free

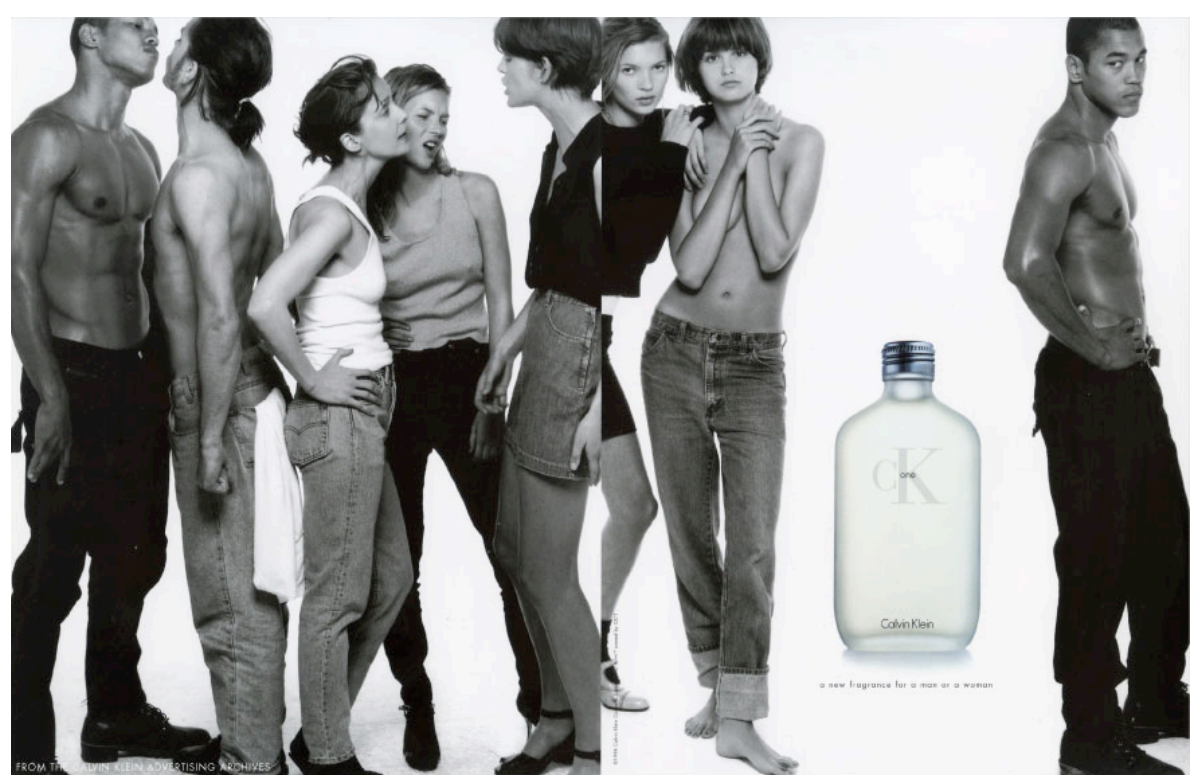

Figura 2.4. Annuncio stampa per cK One. (C) S. Meisel, 1994.

caratterizza per essere molto più statica: ci sono due o tre modelli che guardano direttamente lo spettatore in pose fisse. Come per effetto della presenza del profumo, sembra che le differenze divengano ancora più labili in questa parte dell'annuncio. Nella prima versione (v. fig. 2.3), ad esempio, la modella inginocchiata è Jenny Shimizu: apertamente lesbica e vicina alla comunità delle butch (è stata scoperta mentre lavorava come meccanico a San Francisco), è divenuta una top model proprio grazie a questa campagna, che l'ha resa una

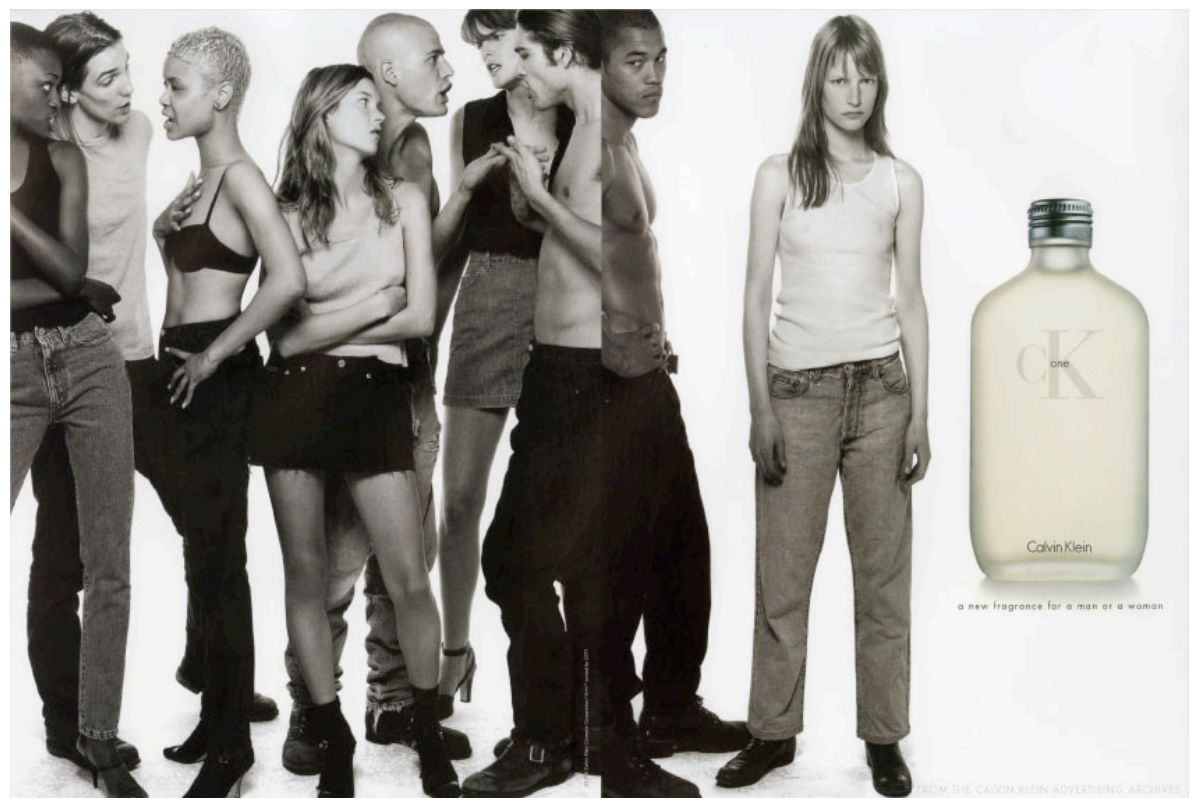

Figura 2.5. Annuncio stampa per cK One. (C) S. Meisel, 1994. 


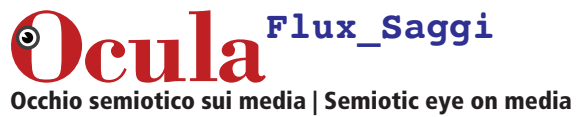

Luca De Bortoli | CK One: a shared fragrance. Corporeità e sessualità gender-free

delle modelle androgine più celebri e rappresentative della categoria. Capelli corti, tatuaggio alla marinara, cinturone e anfibi maschili le conferiscono un tratto di virilità, così come l'essere scoperta dalla vita in su. Nella seconda variante (v. fig. 2.4), Kate Moss è appoggiata a una modella dai capelli corti, che sembra indossare solo un paio di jeans, ${ }^{18}$ così come il modello che appare a destra del flacone. Lo stesso modello appare infine nella terza variante (v. fig. 2.5), a fianco di una modella visibilmente senza reggiseno e in canottiera da uomo, con i soliti jeans e scarpe quasi da lavoro.

A favorire lo smussamento totale delle differenze contribuisce la headline, che recita «A new fragrance for a man or a woman». Se trattassimo di logica classica, diremmo che quella "or" è una disgiunzione inclusiva: $\mathrm{cK}$ One, cioè, è un profumo che può essere indossato da un uomo, da una donna, o da entrambi, poiché il genere di chi lo indossa non fa testo.

\subsection{Videoclip}

Come l'annuncio a stampa, anche il videoclip a supporto della campagna porta la firma di Meisel. Sono diversi i videoclip originari realizzati tra il 1994 e il $1995,{ }^{19}$ e hanno tutti la stessa struttura sintattica, che possiamo pensare come l'espansione cinematica degli annunci stampa. Come in un nastro, sfilano davanti allo spettatore alcuni quadri o tableaux autonomi, ${ }^{20}$ tanto che alcune figure sono tagliate dai confini del tableau successivo (proprio come nell'annuncio): nelle varie scene sono ritratti coppie che si baciano, gruppetti che ballano, altri che discutono, modelli che sorridono e altri che guardano il pavimento, il tutto mentre una voce recita: «The sexy one. The nasty ${ }^{21}$ one. The wild one. The male one. The female one». A questo punto arriviamo all'ultimo tableau, in cui compare Jenni Shimizu: è lei a dire «CK One», o meglio, ad evocare il profumo: pronunciate le parole, la modella svanisce, mentre compare il packshot, e la voce fuori campo conclude: «A fragrance for everyone. From Calvin Klein». ${ }^{22}$

Vengono riprese tutte le analogie formali con l'annuncio: il videoclip è girato in bianco e nero, con l'eccezione del packshot che assume una colorazione virata sul grigio-giallo paglierino alla fine dell'annuncio. I modelli rappresentati vestono nello stile grunge (jeans, canotte, acconciature raffazzonate) di cui sopra. Vedremo nel capitolo successivo (v. 3.1) quale è stata la nuova rappre-

18 Probabile rimando intertestuale: un famosissimo spot per cK Jeans del 1981 (ma che è stato trasmesso per tutti gli anni Ottanta) riprendeva Brooke Shields che diceva: «You want to know what comes between me and my Calvins? Nothing».

19 Per la nostra analisi, come per gli annunci, ci riferiamo al videoclip conservato nell'archivio ufficiale online di Calvin Klein, disponibile all'indirizzo: < https://explore. calvinklein.com/ en_US/timeline/1994/>. Ultima consultazione: 4 giugno 2017.

20 Per una maggiore chiarezza e più facile visualizzazione, abbiamo spezzato lo spot nei vari tableaux. Vedi l'Appendice in calce.

21 Una possibile traduzione di nasty è "nauseante", che per una pubblicità di profumi è assolutamente ironica e inedita.

22 Leggera variazione della headline, che compare comunque in sovraimpressione. In altri spot viene mantenuta quella dell'annuncio stampa. 


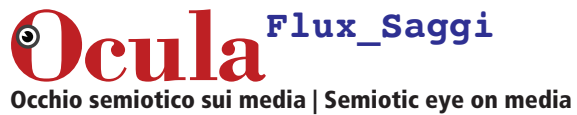

Luca De Bortoli | CK One: a shared fragrance. Corporeità e sessualità gender-free

sentazione dei corpi e concezione della sessualità che ha completamente distinto questa campagna dalle precedenti; mettiamo intanto in luce un aspetto originale relativo ai modelli scelti. Di fatto, solo Kate Moss è una top model (Jenni Shimizu era stata appena notata per caso): tutti gli altri sono non professionisti, ma ragazzi reali. A questo si lega un altro punto di distinzione rispetto alle campagne pubblicitarie della moda: generalmente, infatti, i modelli vengono scelti come rappresentanti di un desiderio per il consumatore, come incarnazione di un /voler-essere/. In genere si tratta di personaggi famosi o super top-model dotati di celebrità o fascino inarrivabile. Tutto il contrario per cK One, in cui la /relazionabilità/, il potersi identificare con i ragazzi della campagna è un valore forte. Proprio a proposito dei modelli, Calvin Klein ha dichiarato di essersi ispirato al mondo della figlia, e di aver voluto modelli reali per raccontare il mondo dell'epoca:

The change has been that we use a lot of real people, and they're not professional models. So the world can see this in the way it really is. And I think that's why people have identified with a lot of our images - because they're very real; there's nothing artificial. [...] So the world is seeing a reflection of what's really going on. I think people get it, and I think they like it. (Kaplan 1995: 52-55)

Tutto il contrario rispetto a campagne pubblicitarie che mitizzano non solo il prodotto, ma anche il testimonial. Nel capitolo successivo mettiamo in luce, attraverso un contrasto con alcune pubblicità più datate, alcune tendenze precedenti alla nostra campagna, per meglio chiarirne la novità e l'originalità. In particolare, vedremo come una rappresentazione dei corpi focalizzata su due poli (quello maschile del macho e quello femminile della femme fatale), sia dipendente da una concezione della sessualità completamente diversa in cK One.

\section{Corpi e sessualità}

Su una particolare rappresentazione dei corpi, e sull'articolazione del senso della sessualità, è incentrata gran parte della campagna. Non solo quelli che vediamo negli annunci e nei videoclip sono corpi di ragazzi e ragazze, ma il senso di una categoria (quella della sessualità), che più in profondità va ad interpolare la rappresentazione del corpo, ne risulta articolato in una maniera inedita e controcorrente.

\subsection{Macho e Femme fatale}

In semiotica il senso si dà per differenza, e risulta facile apprezzare il cambiamento tra un sistema culturale all'altro andando a valutare (in diacronia) quali sono stati i cambiamenti di senso nel modo di pensare, rappresentare e articolare questa tematica. Nell'universo semantico del discorso della moda, noteremo un notevole cambiamento tra quella che era la rappresentazione dei corpi e della sessualità negli anni Ottanta e inizio Novanta rispetto alla nuova proposta scaturita da cK One. Nella cultura della moda degli anni Ottanta, contraddistinta in buona parte da una sensualità edonistica, idealizzata, gla- 


\section{Ocula ${ }^{\text {Flux_saggi }}$ \\ Occhio semiotico sui media | Semiotic eye on media}

Luca De Bortoli | CK One: a shared fragrance. Corporeità e sessualità gender-free

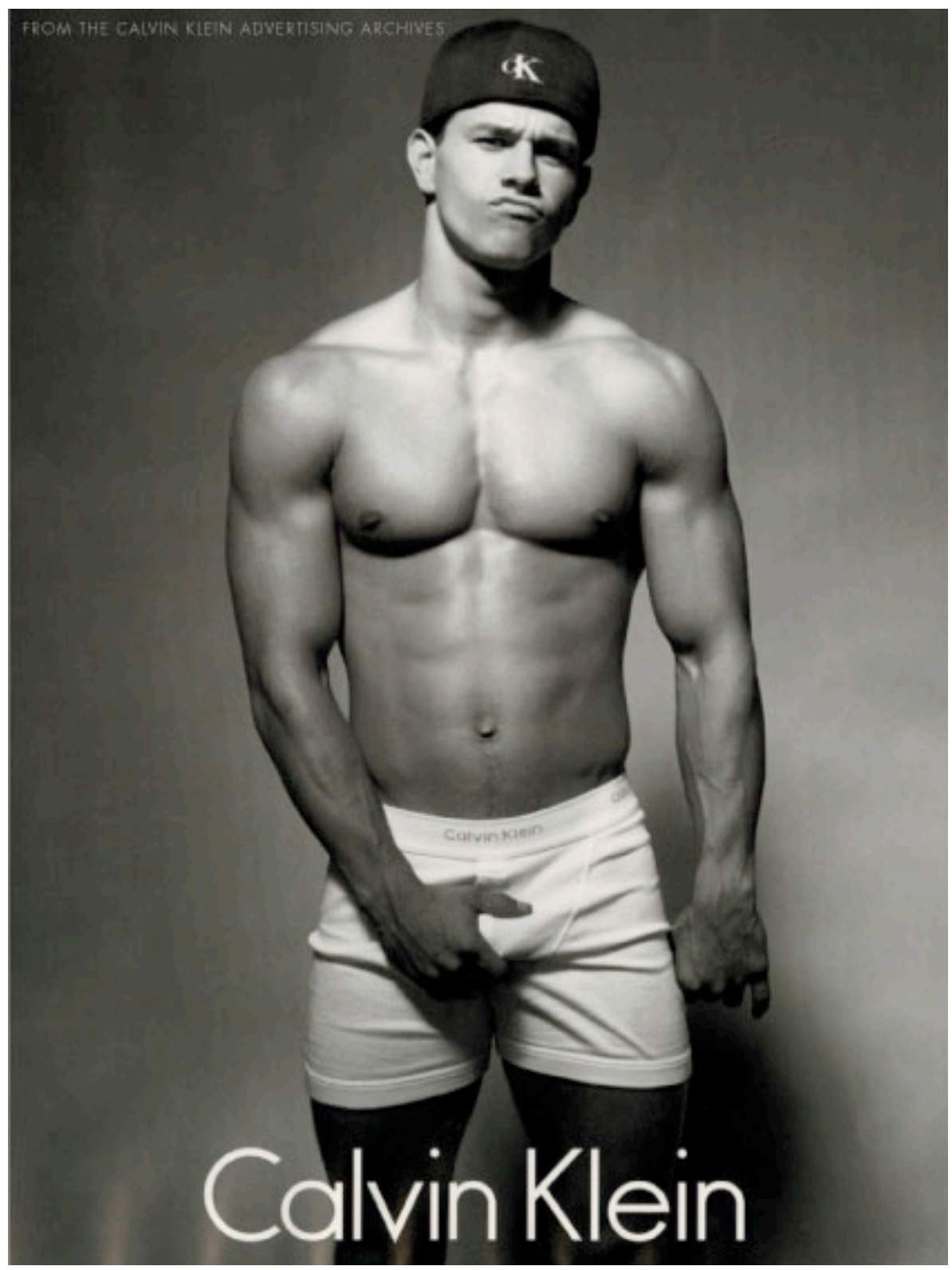

Figura 3.1. Mark Wahlberg per cK Underwear. (C) H. Ribbs, 1992.

mour, polarizzata tra le figure-simbolo del macho e della femme fatale (con le relative incarnazioni corporali), abbiamo una logica di contrarietà, giocata sull'asse maschile/femminile. Una naturale discorsivizzazione, nell'ambito delle pubblicità di profumi, risulterà in una settoriale divisione tra profumi maschili e profumi femminili, senza soluzione di continuità tra i due: impossibile per un uomo indossare Chanel $n^{\circ} 5$, così come per una donna indossare Eau Sauvage di Dior. Da qui l'esigenza di avere un testimonial del sesso corrispondente, con cui l'enunciatario possa identificarsi (ed eventualmente, a cui la boccetta possa assomigliare). Anche la separazione del mercato ne è una conseguenza diretta. 


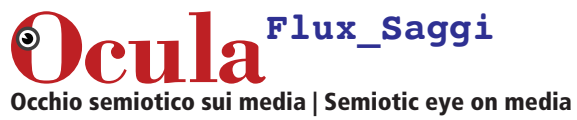

Luca De Bortoli | CK One: a shared fragrance. Corporeità e sessualità gender-free

La genderizzazione corrispondente, dunque, sarà magnificata: le donne ritratte sono sensualissime, erotiche, fatali: hanno acconciature fluenti, indossano vestiti ricercati e hanno forme evidenti. Gli uomini sono vigorosissimi, padroni e seduttori, virili e potenti.

Restando nell'universo Calvin Klein, vediamo la differenza con altre due campagne pubblicitarie precedenti a cK One, per mettere in contrasto la rappresentazione del corpo maschile e femminile e la successiva transizione verso cK One. La prima (v. fig. 3.1) è cK Underwear con Mark Wahlberg (1992), e porta la firma di Herb Ritts, storico esponente della glamour-photography degli anni Ottanta e Novanta. All'epoca Mark Wahlberg era noto in USA come "Marky Mark", e si dedicava al rap e all'hip-hop. Rappresentato in questo annuncio, notiamo la presenza di un berretto indossato al contrario (o una bandana), l'espressione fiera e aggressiva di sfida, e la mano che stringe le pudenda. Sono caratteristiche che ci riportano a una cultura urbana, di ghetto, in cui la vince il più forte e in cui vige la logica dell'alpha-male. Wahlberg ne è un tipo: ci appare aggressivo e compiaciuto. Il corpo, ovviamente, è ipersessualizato, indice di una virilità ostentata e tronfia. La rappresentazione segue un trend che negli ultimi anni è tornato con forza alla ribalta delle riviste patinate. ${ }^{23}$

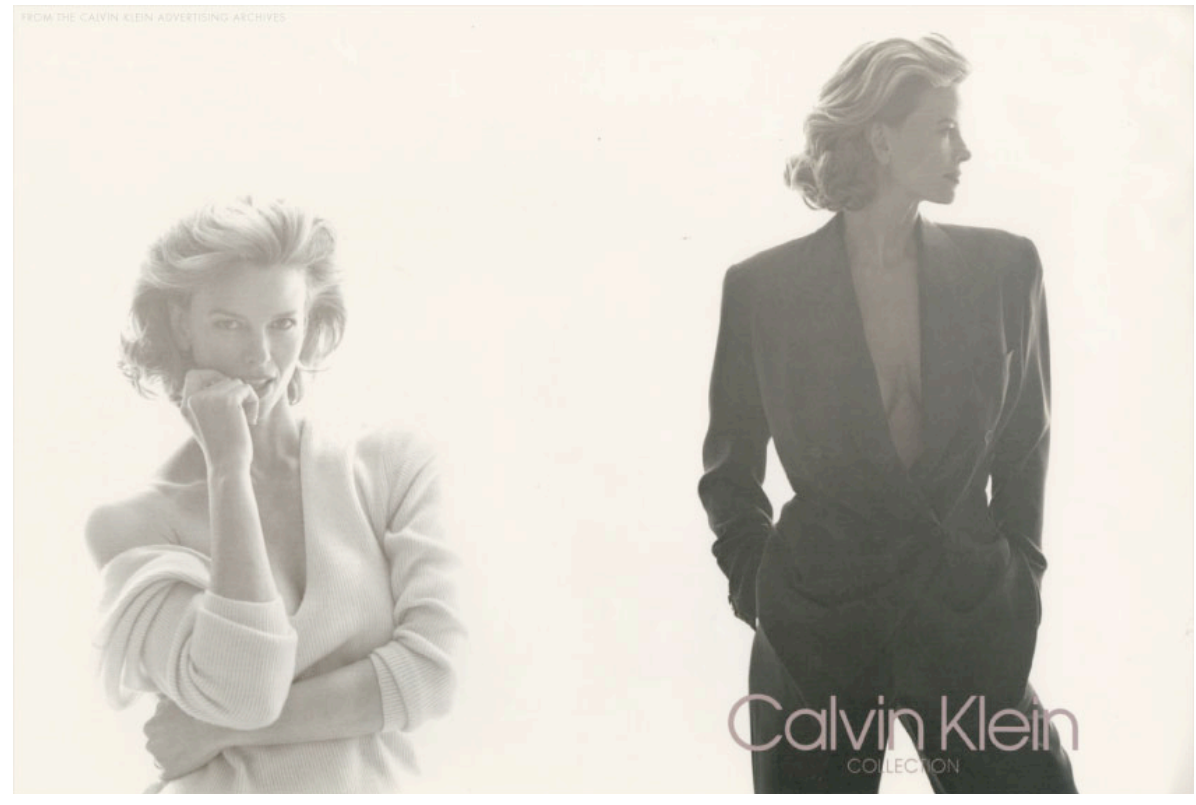

Figura 3.2. Lisa Taylor per Calvin Klein. (C) S. Meisel, 1994.

23 Con esiti quasi parossistici. Quello di Wahlberg è, tutto sommato, il suo di corpo, e l'ipersessualizzazione riguarda la scelta di un modello culturale (quello del 'macho' o alpha-male del ghetto), non basato solamente sulla corporeità, ma anche sull'atteggiamento. Come nota Cosenza (2010), al pari di quanto avviene con le donne, una tendenza nella rappresentazione del corpo maschile degli ultimi anni è quella che ricerca l'irrealtà e la plastificazione. Interessante notare la campagna, sempre cK Underwear, con testimonial Justin Bieber (2015). Pur facendo eco a quella di Marky Mark, Bieber ha un'espressione molto più vuota; il suo corpo, inoltre, è stato massicciamente photoshoppato, rendendolo come un manichino o un bambolo 


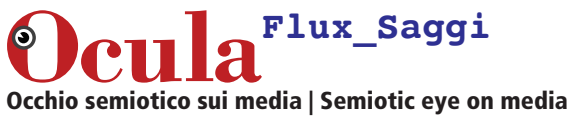

Luca De Bortoli | CK One: a shared fragrance. Corporeità e sessualità gender-free

Vediamo ora l'esempio femminile, in questa campagna con Lisa Taylor (v. fig. 3.2). Siamo nel 1992, e l'obiettivo fotografico è quello di Meisel, che realizzerà poi la campagna per $\mathrm{cK}$ One.

Qui la femminilità riprende stilemi tipici degli anni Ottanta: glamour, con una certa aria di vaghezza, eleganza ed erotismo, dato dalla spallina del vestito della sinistra. C'è da dire che, effettivamente, la Taylor appare già più androginizzata nell'immagine di destra (l'abito con pantalone sembra essere ispirato a un completo da uomo), ma né l'abito, né lo sguardo scostante nascondono il seno o la chioma fluente. Entrambe le immagini sembrano essere contornate come da un'aura di sensualità ed estetismo. Il tipo a cui fa riferimento è certamente la femme fatale. ${ }^{24}$

Punto forte di distinzione è quindi la /relazionabilità/, totalmente assente in queste campagne precedenti che giocano invece sul /voler essere/ forti e virili come Wahlberg o charmant e bellissime come la Taylor. CK One ha quindi rappresentato una rivoluzione nella ricerca di un profumo veramente universale (grazie al fatto di essere unisex) e vicino al mondo reale, come reali sono i modelli scelti da Calvin Klein.

Nell'analisi della campagna di cK One abbiamo ipotizzato l'utilizzo della prova di commutazione tra generi, concludendo che non avrebbe creato scompensi di senso significativi nell'economia generale. Tutto il contrario in questo caso, in cui arriveremmo quantomeno a suscitare il comico: basti immaginare Lisa Taylor nella stessa espressione e posa (gesto della mano incluso) di Marky Mark. Questo avviene per un motivo specifico, di seguito illustrato.

La rappresentazione dei corpi (in questi due casi) dipende da uno specifico sistema, che è quello della sessualità e dal modo in cui questa categoria viene percepita. Pensando ai corpi in maniera binaria (con ripercussioni ad esempio sul mercato, in cui vengono create due linee di profumi), il risultato consiste in una polarizzazione tra i due generi: maschi super-virilizzati e donne super-femminilizzate, spesso attraverso tutti gli stereotipi e tipi culturali del caso. Di fatto, possiamo affermare che il paradigma della moda degli anni Ottanta e inizio Novanta si svolge per contrapposizione tra i termini contrari ${ }^{25}$ della categoria /maschile vs femminile/, in cui solo uno dei due viene attivato: ne è una prova la polarizzazione dei termini, così come l'impossibilità di commutarli.

In cK One il procedimento di senso è basato sulla messa in parentesi di questa opposizione, che diventa non pertinente: non è importante il genere di chi compra il profumo, perché è un profumo universale e per tutti. Prove di commutazione ci convincono del fatto che la differenziazione sessuale

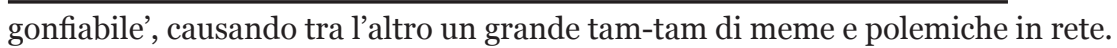

24 Qualcuno potrebbe azzardare il modello in Marlene Dietrich, per via della somiglianza fisica e per l'abbigliamento.

25 La tentazione di resuscitare il quadrato semiotico della sessualità è forte, ma serve grande cautela. Accogliamo in pieno le critiche che sono state mosse al "dispositivo organizzatore del senso" (in particolare, cfr. Paolucci 2010: cap. 3), e infatti l'uso che vorremmo farne ha un solo carattere illustrativo e topologico - e non operativo (nel senso sintattico di passaggi o operazioni da un termine all'altro). 


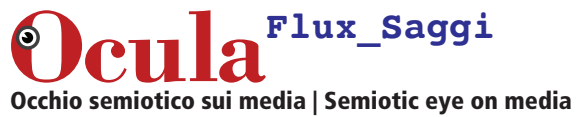

Luca De Bortoli | CK One: a shared fragrance. Corporeità e sessualità gender-free

tradizionale e binaria ${ }^{26}$ non sia pertinente per la filosofia $\mathrm{cK}$ One, che si gioca di fatto su un'altra logica, in qualche modo più ampia, e non relegata esclusivamente alla sessualità. Come già notato a pochi mesi di distanza dal lancio della campagna «cK One è diventato un caso nazionale, lanciato come un'eau de zeitgeist trasversale agli stereotipi sessuali» (Di Drusco 1995: 58; c.n.).

Quello che è un valore profondo importante è in effetti quello della universalità attraverso la /condivisione/ («a shared fragrance») e progressiva inclusione: la headline della campagna recitava «a fragrance for a man or a woman», che viene a sostituirsi con "A fragrance for everybody» nel videoclip. La nuova campagna del 2001 avrà poi come headline: «For all. For ever».

\subsection{Il caso Altered States}

In uno spot intitolato Altered States ${ }^{27}$ abbiamo un caso ancora diverso. Lo spot mostra la trasformazione da $\mathrm{cK}$ One a $\mathrm{cK}$ Be (di fatto, cambiano solo il colore del flacone, nero opaco, e alcune note di fragranza). Ma la differenza più evidente - almeno per chi è sprovvisto di narici allenate - si situa proprio a livello della rappresentazione del corpo e della sessualità.

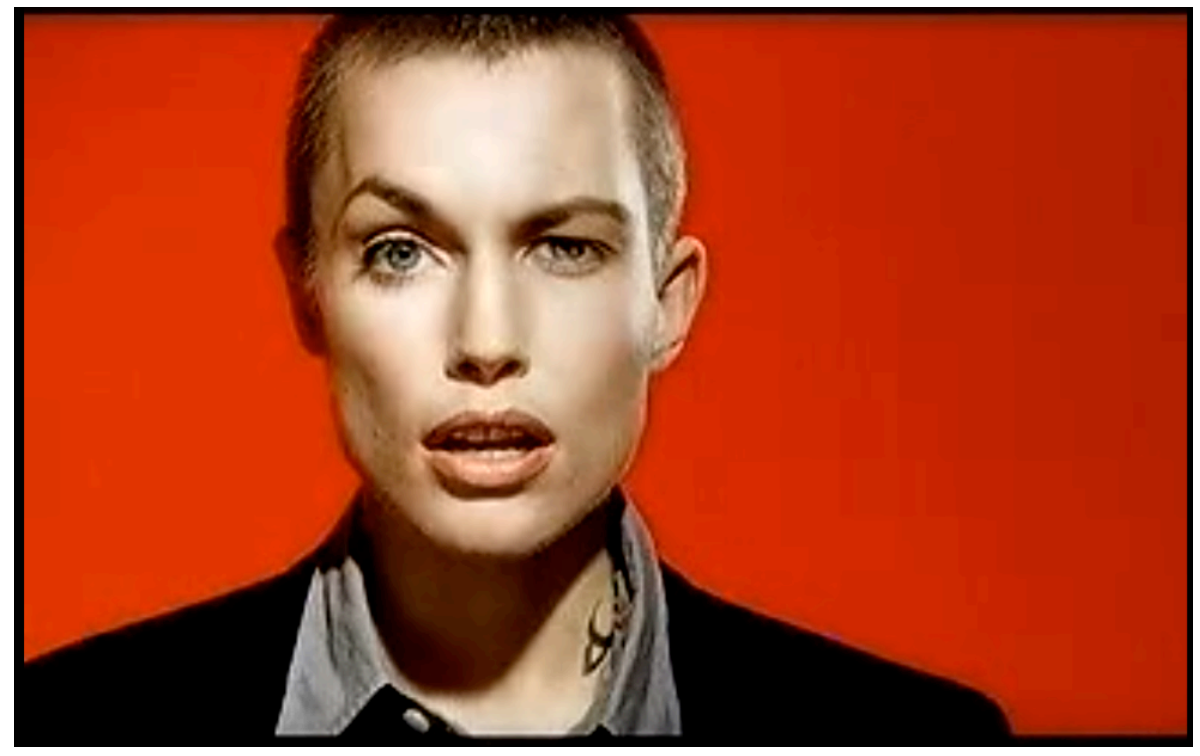

Figura 3.3. Yon Yonson nello spot Altered States.

26 Non dimentichiamo che ogni fatto di cultura è inter-relato nel macro-sistema enciclopedico della semiosfera: la campagna cK One viene emessa nel 1994, mentre pochi anni prima, T. de Lauretis curava il numero speciale della rivista «Differences» intitolandolo Queer Theory: Lesbian and Gay Sexualities (1991), seguito a ruota da Bodies that Matter (1993) di J. Butler e da innumerevoli lavori di altri accademici che sono poi divenuti nomi famosi della Queer Theory.

27 Visualizzabileall'indirizzo: <https://www.youtube.com/watch?v=BKvjZUAPve4>. Ultima consultazione: 13 giugno 2017. Informazioni riguardo il regista dello spot e l'anno di produzione non risultano disponibili. 


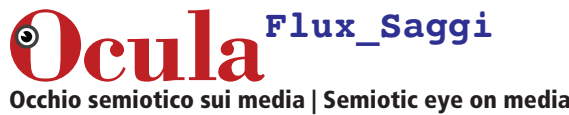

Luca De Bortoli | CK One: a shared fragrance. Corporeità e sessualità gender-free

Di fronte alla telecamera, abbiamo un individuo (v. fig. 3.3) che recita una filastrocca ricorsiva ${ }^{28}$ (presente tra l'altro in Mattatoio $n^{\circ} 5$ di Kurt Vonnegut). Lo chiamiamo individuo, poiché non pare sessualmente determinato, ed è su questa indeterminatezza e contrasto che si gioca lo spot. Yon (così ci riferiremo al protagonista) presenta un tipico look androgino, il cui possibile modello, o ispirazione, potrebbe essere Annie Lennox in Sweet Dreams (are made of this): non solo, infatti, l'outfit è identico (giacca e camicia, capelli corti) e la somiglianza del viso è grande, ma altri elementi (quali lo sfondo rosso, o l'insistenza nel riprendere gli occhi e la bocca di Yon) ce lo fanno ben supporre.

Non è solo l'apparenza. Dopo aver pronunciato le prime parole, ci rendiamo conto dell'irrequietudine e instabilità di Yon, che sembra governato da due forze interne e contrarie: i suoi occhi, infatti, si muovono indipendentemente l'uno dall'altro, come se cercassero l'uno di invadere il territorio antagonista, o di emergere del tutto prendendo il controllo di Yon. È al minuto o:12 che la voce di Yon cambia completamente, e diventa la voce che siamo abituati a riconoscere come femminile. Infine, un frammento visivo (v. fig. 3.4), inserito al minuto 0:23, ci raffigura il viso di Yon tagliato tra le due forze:

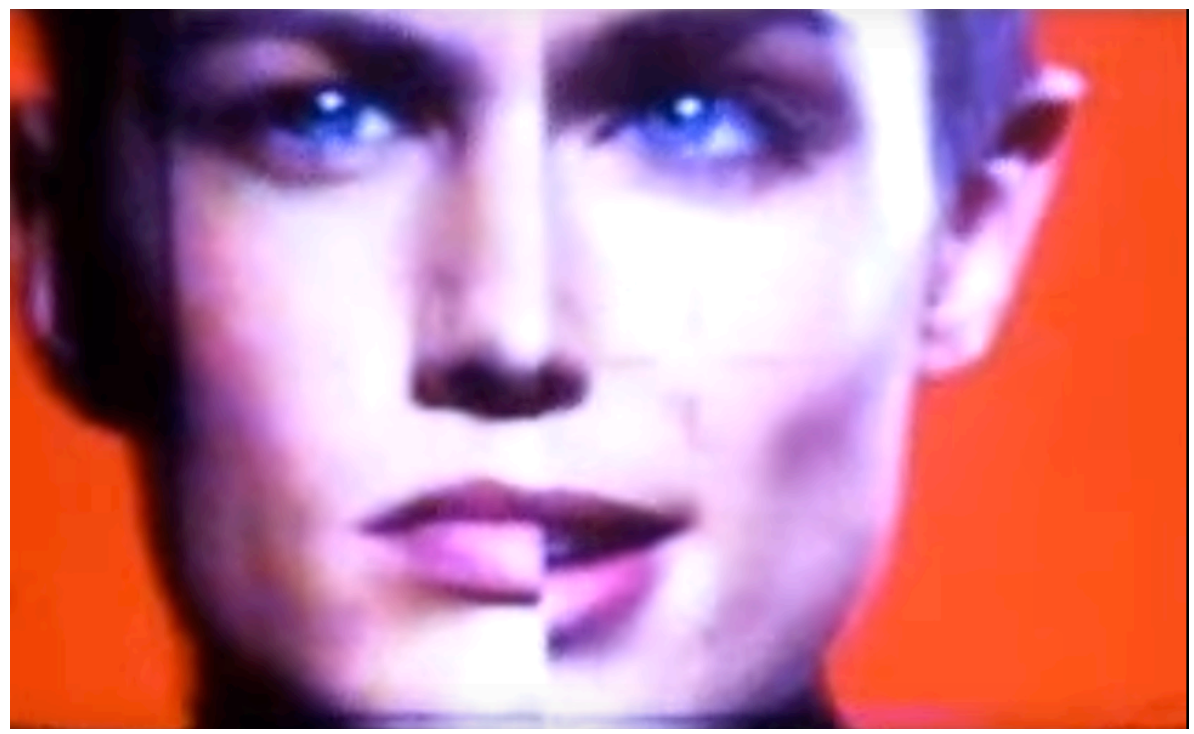

Figura 3.4. Fotogramma al minuto $0: 23$

Un'analisi limitata a questo spot prenderebbe senz'altro in considerazione il ricorrere a quella porzione di enciclopedia che ha a che fare con la fantascienza e le distopie; ${ }^{29}$ ma tralasciamo questo aspetto, per mettere in relazione più generale questo spot con le altre campagne di Calvin Klein. Siamo distanti

28 Questo il testo: «My name is Yon Yonson / I come from Wisconsin / I work in a lumber mill there / When I walk down the street / the people I meet / say: 'Hey! What's your name?' / And I say: 'My name is Yon Yonson / I come from Wisconsin / I work in a lumber mill there...».

29 Non solo il rimando al romanzo di Vonnegut, ma anche il titolo dello spot, Altered States, richiama l'omonimo film di fantascienza di Ken Russell (1980). 
Luca De Bortoli | CK One: a shared fragrance. Corporeità e sessualità gender-free

anni luce dallo spot per cK One. Lo shift, oltre ad avere divergenze in fatto di estetica e gusto, rivela un diverso investimento valoriale e semantico della sessualità. Tra i due spot è questa una grandezza su cui possiamo rendere comparabili e confrontabili i due testi.

In cK One le differenze tra i generi venivano via via smussate e rese non pertinenti in nome dell'unità e della inclusività, in un Uno armonico, in cui chiunque avrebbe potuto trovare espressione: da qui l'utilizzo di decine di modelli dai corpi differenti. In cK Be è l'esatto opposto: la presenza di entrambe le componenti in un unico individuo (le diverse tonalità della voce, le opposte direzioni dello sguardo, la segmentazione del viso) figurativizzano un contrasto inconciliabile, fondato su una relazione irriducibile tra i termini: Yon ci appare infatti come un essere contrastato, irrequieto, combattuto tra i due principi. Il fotogramma inserito nel video è quello che meglio visualizza il contrasto, ma anche l'autonomia degli occhi ne è un segno inequivocabile. La sessualità è qui il terreno di battaglia tra due forze opposte e contrarie, che tuttavia non possono stare assieme. In $\mathrm{cK}$ One, invece, è proprio in virtù di un'uguaglianza e armonia di status che la condivisione è possibile.

\section{Conclusioni}

L'analisi si è concentrata su una delle campagne più famose della storia della moda degli anni Novanta, che ha profondamente inciso il mercato cosmetico e il marketing dei fashion brands. CK One ha rappresentato indubbiamente un punto di rottura con una concezione separatista dei corpi (maschile/ femminile), che vuole polarizzare il primo sul modello del macho o dell'uomo che non deve chiedere, conquistatore e virile, e il secondo sulla femme fatale, sensuale, sfuggente e inarrivabile.

Nell'assiologia di cK One, il corpo non si trova conteso tra le due pretese in larga parte stereotipizzanti. La possibilità di essere un'essenza «For a man or a woman» o di essere «For all, for ever» apre tutti gli scenari possibili, e soprattutto quelli già disponibili ai consumatori, cioè i propri: cK One è riuscito infatti a calare i propri valori dal livello virtuale del /voler essere/ a quello reale dell'identificazione, possibile anche grazie a fattori di prossimità culturale (modelli non professionisti, abbigliamento casual, situazioni di relazioni quotidiane, cultura musicale affine ecc.).

Abbiamo riscontrato questa assiologia in tutti i livelli di analisi, da quello sensoriale-olfattivo (che ha messo in luce la neutralità della fragranza, non connotata come femminile o maschile) a quello semantico (secondo la particolare concezione e categorizzazione della sessualità).

Pur essendo un prodotto che per ispirazione e prossimità culturale è stato avvicinato alla cultura giovanile - che tra l'altro, per la prima volta trovava una campagna in cui rispecchiarsi, e non una mitizzante - con l'andare del tempo, cK One ha perso quell'aura più trasgressiva, divenendo però così sempre più universale, e conquistando le più variegate fasce di consumatori. 
Ocula ${ }^{\text {Flux_saggi }}$

Occhio semiotico sui media | Semiotic eye on media

Luca De Bortoli | CK One: a shared fragrance. Corporeità e sessualità gender-free

\section{Appendice}

Visualizzazione sintagmatica dello spot cK One. (C) S. Meisel, 1994
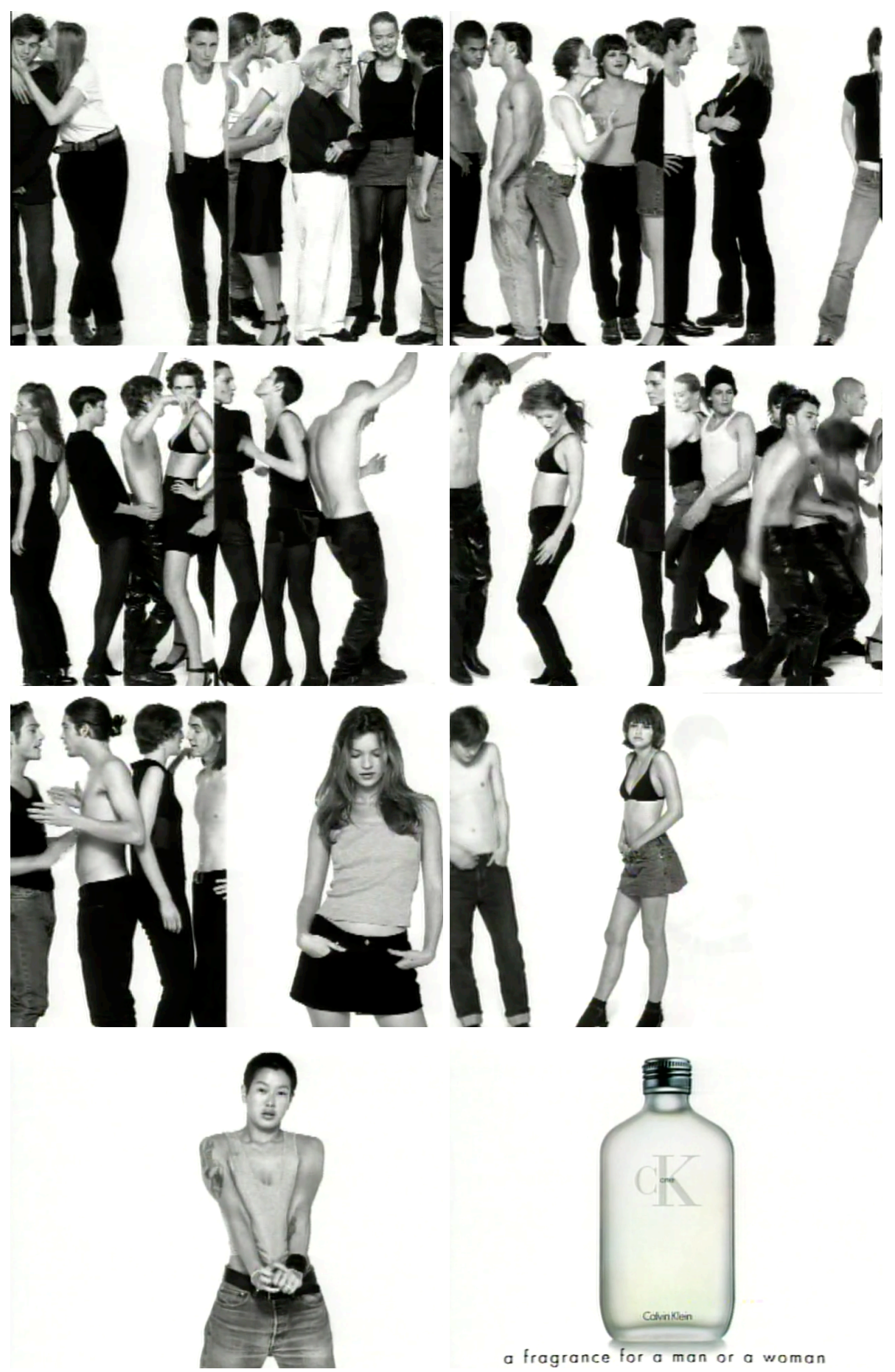

a fragrance for a man or a woman 


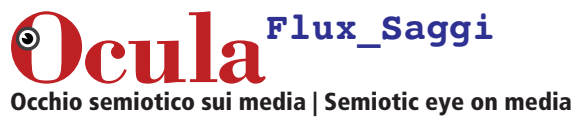

Luca De Bortoli | CK One: a shared fragrance. Corporeità e sessualità gender-free

\section{Bibliografia}

Cavalieri, Rosalia

2010 "Nasus cogitans. Divagazioni sull'intelligenza del naso", Dialeghestai, a. XII, agosto 2010

Cosenza, Giovanna

2010 "Il corpo degli uomini”, Alfabeta 2, 4, novembre 2010.

Di Drusco, Fabia

1995 "Idem senitre. Scent for two", Vogue, 554 (dicembre 1995), p. 58.

Jakobson, Roman O.

1959 "On Linguistic Aspects of Translation", in Brower, R. A. (a cura di), On translation, Cambridge, Harvard UP, pp. 232-9.

Kaplan, James

1995 “The Triumph of Calvinism”, The New York Magazine, 4, 19 September 1995, pp. 47-57.

Leroy-Gouran, André

1997 "Basi corporee dei valori e dei ritmi”, in Id., Il gesto e la parola, in 2 voll., vol. II: "La memoria e i ritmi”, Torino, Einaudi, pp. 328-47.

Ostrom, Lizzie

2015 Perfume: a Century of Scents, London, Hutchinson.

Paolucci, Claudio

2010 Strutturalismo e interpretazione. Ambizioni per una semiotica a minore, Milano, Bompiani.

Poucher, William A.

2000 Perfumes, Cosmetics and Soap, ed. by H. Butler, Dordrecht, Kluwer Academic.

Strauss, William; Howe, Neil

1991 Generations: The History of America's Future, 1584 to 2069, New York, Harper Perennial.

Luca De Bortoli è laureato cum laude in Lingue e Letterature Straniere. È stato studente presso l'Università di Tartu e l'Università Statale di San Pietroburgo, dove ha potuto lavorare alla delineazione della figura di Ju. M. Lotman come settecentista. Sta compiendo gli studi magistrali in Semiotica presso l'Università di Bologna. 